\title{
Multiple Ossicular Dislocation Including Stapediovestibular Dislocation Presenting with Conductive Hearing Loss
}

\author{
Geonho Lee ${ }^{1}$, Yoonjoong Kim ${ }^{1}$, and Bong Jik Kim² \\ ${ }^{1}$ Department of Otorhinolaryngology-Head and Neck Surgery, Chungnam National University Hospital, Daejeon, Korea \\ ${ }^{2}$ Department of Otolaryngology-Head and Neck Surgery, Chungnam National University College of Medicine, \\ Chungnam National University Sejong Hospital, Sejong, Korea
}

\author{
Received May 25, 2020 \\ Revised August 17, 2020 \\ Accepted October 6, 2020
}

Temporal bone trauma can cause hearing loss and in case of prolonged conductive hearing loss, traumatic ossicular injury should be considered. Separation of the incudostapedial joint is the most common lesion, and stapediovestibular dislocation is relatively rare but can easily cause perilymphatic fistula. Here, we report a very rare case of external stapediovestibular dislocation after trauma, ending up with successful surgical outcome. A 27 -year-old man with non-progressive hearing loss on the right side since childhood visited the clinic. Audiogram showed a conductive hearing loss with air-bone gap of $55 \mathrm{~dB}$ on the right side. Temporal bone CT revealed the disruption of ossicular chain. An exploratory tympanotomy identified multiple ossicular disruptions including external stapediovestibular dislocation with shiny fibrous membrane sealing the oval window. Ossicular chain reconstruction was performed using the total ossicular replacement prosthesis of titanium. A postoperative audiogram showed a recovery of air-bone gap less than $10 \mathrm{~dB}$. To the best of our knowledge, this is the first case of external long-standing stapediovestibular dislocation, with oval window completely sealed with fibrous membrane, ending up with successful hearing recovery by surgery. This case would help dealing with such condition which can be encountered in the clinic.

J Audiol Otol 2021;25(3):159-162

KEY WORDS: Trauma · Conductive hearing loss · Ossicular dislocation · Stapediovestibular dislocation · Fibrous membrane.

\section{Introduction}

Trauma to the temporal bone usually manifests as sensorineural, conductive, or mixed hearing loss. Conductive hearing loss after head trauma generally results from a tympanic membrane perforation, hemotympanum, or ossicular disruption. In a case of persistent conductive hearing loss of $30 \mathrm{~dB}$ or more that lasts longer than 2 months after the tympanic membrane has healed, ossicular disruption should be considered.

Traumatic ossicular injury can be classified into the following categories: incudostapedial separation, incudomalleolar separation or dislocation, dislocation of the malleus or incus, stapediovestibular dislocation, and fracture of the ossicles

This is an Open Access article distributed under the terms of the Creative Commons Attribution Non-Commercial License (https:/creativecommons.org/licenses/by-nc/4.0/) which permits unrestricted non-commercial use, distribution, and reproduction in any medium, provided the original work is properly cited.
[1-3]. In literature reviews, the incus was the most commonly involved ossicle with incudostapedial joint separation as the most common location of traumatic injury; external stapediovestibular dislocation was extremely rare [4].

Here, we report a case showing the natural course of longstanding external stapediovestibular dislocation following a traffic accident that presented with conductive hearing loss, which was successfully treated with surgery.

\section{Case Report}

A 27-year-old man with a 22-year history of non-progressive hearing loss on the right side visited our tertiary otology clinic with the goal to recover his hearing for his next career as an air force officer. He had been in a traffic accident at the age of five and presented with bloody otorrhea; he was admitted to the hospital for a few days. Since 22 years have passed 
already, the patient and his parents could not remember the accident exactly. The accident was a pedestrian traffic accident hit by a truck, and he lost consciousness immediately after the accident, but there was no major trauma in other parts. Although the exact onset was unclear, hearing loss seemed to have existed since then. He could not remember whether he had vertigo at that time.

An audiologic assessment demonstrated conductive hearing loss with a pure-tone average of $62 \mathrm{~dB} \mathrm{HL}$ and an air- bone conduction gap of $55 \mathrm{~dB}$ on the right side, with preservation of bone conduction hearing (Fig. 1A). An otoscopic examination showed an intact tympanic membrane with slight displacement of the malleus handle and temporal bone CT revealed disruption of the ossicular chain involving all ossicles (the malleus, incus, and stapes) (Fig. 2).

To increase the possibility of improving his hearing, an exploratory tympanotomy was performed. The surgery was performed with a retroauricular approach. When the tympano-
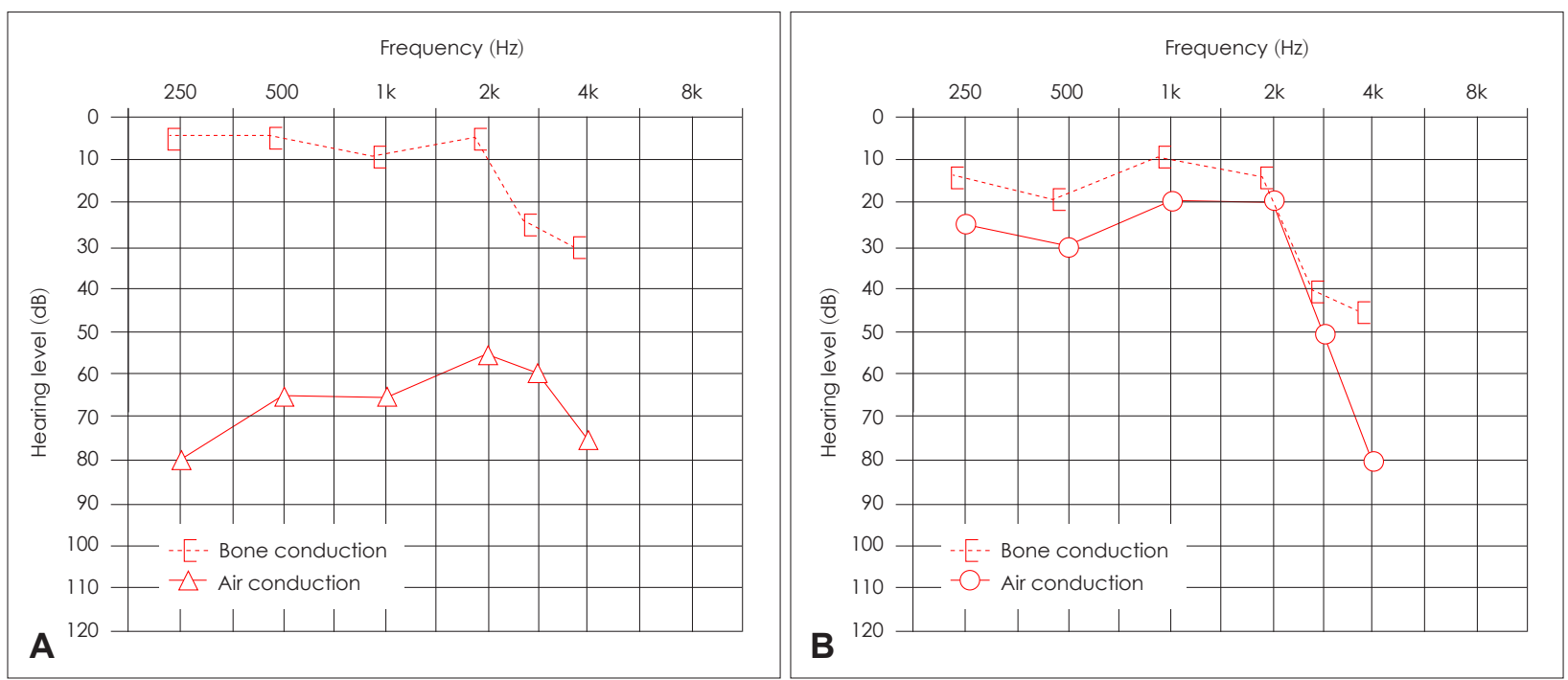

Fig. 1. Preoperative and postoperative audiogram of the patient. (A) Preoperative pure tone audiogram of the right side showing conductive hearing loss with an air-bone gap of more than $50 \mathrm{~dB} \mathrm{HL}$. (B) Postoperative pure tone audiogram of the right side showing improved hearing.
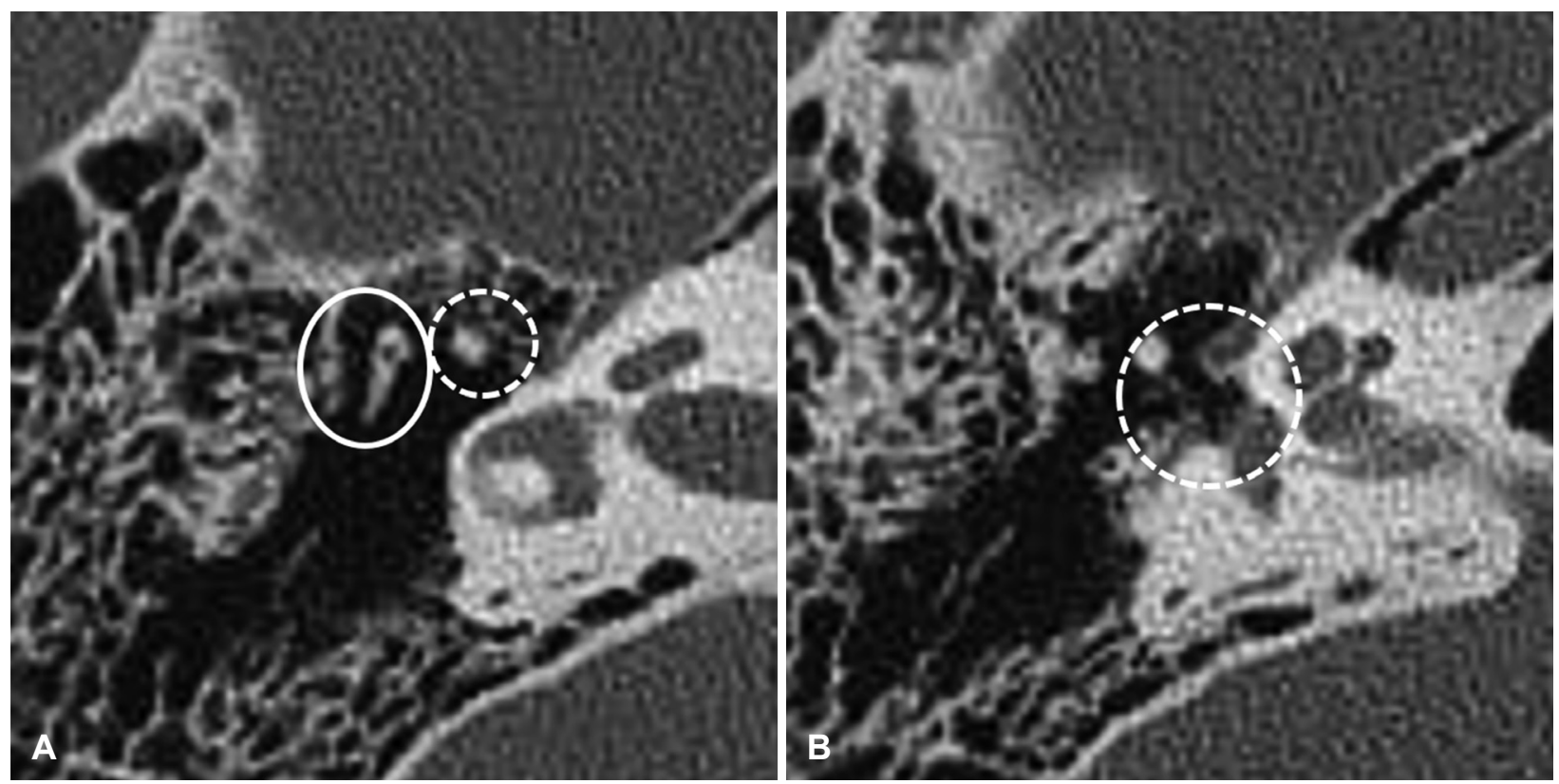

Fig. 2. Preoperative temporal bone CT images. (A) Preoperative temporal bone CT image showing an incudomalleolar separation. The dashed circle and solid circle indicate the dislocated malleus and incus, respectively. (B) Suspicious external stapediovestibular dislocation: the dotted circle indicates an externally dislocated stapes from the vestibule. 
meatal flap was elevated and the middle ear was observed, the displaced malleus and the stapes were observed, and the stapes were completely rotated (Fig. 3A). The displaced stapes had severe adhesion, and the incus was not visible. Due to the limited surgical field of view, we decided to accurately check the ossicle by approaching through the mastoid antrum. When observed through the antrum, the malleus and the incus were separated, the malleus was on the medial side of the incus, and adhered to the stapes by fibrotic band (Fig. 3B-D). Fracture of the ossicles was not observed and the displaced incus was removed. When the displaced stapes were removed and the oval window was observed, the foot plate and annular ligament were replaced by a shiny, rubbery fibrous membrane (Fig. 3E, F). To restore the ossicular chain, enabling efficient sound transmission, a piece of cartilage from the cavum concha was trimmed to fit into the space of the missing footplate and a total ossicular replacement titanium prosthesis (TORP; $4.0 \mathrm{~mm}$ ) with a cartilage interface was positioned between the preinserted cartilage and the tympanic membrane and secured with soft tissue, gelfoam, and fibrin glue. The patient did not complain of any surgical complications and a follow-up audiogram showed recovery of an air-bone gap less than $10 \mathrm{~dB}$ with subjective satisfaction (Fig. 1B).

\section{Discussion}

External stapediovestibular dislocation is a very rare condition of ossicular injury that can occur after temporal bone trauma and can lead to vestibulocochlear symptoms in addition to conductive hearing loss. Given the preoperative evaluation and intraoperative findings, our hypothetical mechanism of ossicular dislocation was as follows: the blunt force trauma caused the weakest part, incus, to separate from the malleus and stapes, and then the intact stapes and footplate were dislocated from the oval window. The oval window may have been sealed immediately with blood from the surrounding structures, which filled the middle ear space, possibly preventing a perilymphatic fistula (PLF). As another hypothesis, we thought that the dislocation of the ossicle would have occurred due to the indirect force caused by acceleration-decel-
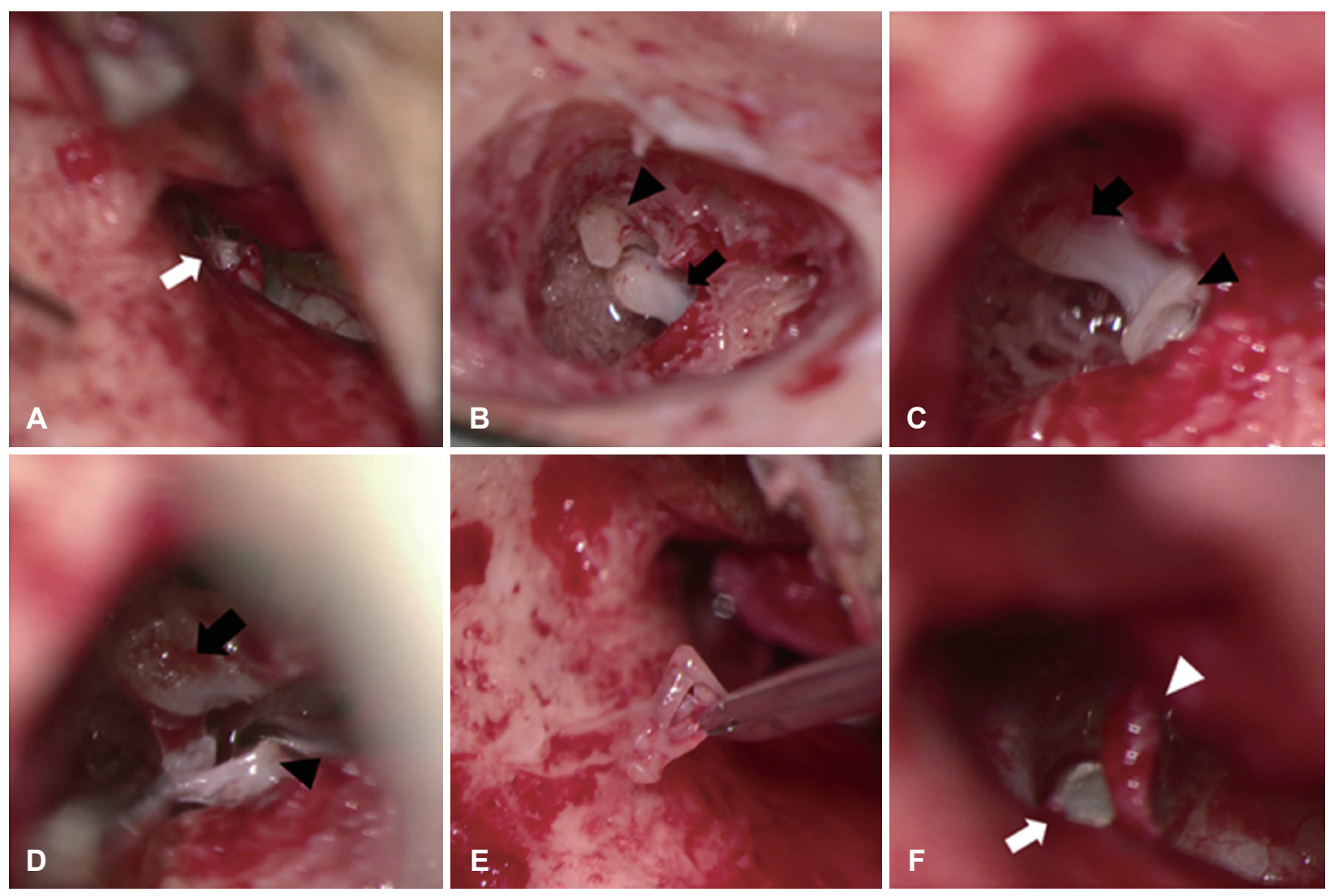

Fig. 3. Intraoperative findings. (A-D) Intraoperative findings showing dislocated ossicles. (A) The displaced stapes (white arrow) showing adhesions with surrounding mucosa: view through the ear canal. (B) The dislocated incus (black arrowhead) and malleus (black arrow): view through the antrum. (C) The displaced malleus (black arrow) and stapes (black arrowhead) connected by fibrotic band after removal of the incus: view through the antrum. (D) The head of malleus (black arrow) and stapes footplate (black arrowhead) after removal of fibrotic band. (E) Removal of the dislocated stapes with an intact footplate. (F) The shiny fibrous membrane (white arrow) replacing the oval window. The white arrowhead indicates the chorda tympani nerve. 
eration [5].

This is the first reported case showing the natural course of long-standing trauma-induced conductive hearing loss caused by spontaneous sealing off of presumed leaked perilymph from the oval window that was successfully treated surgically.

Since the stapes' location deep in the tympanic cavity normally protects it from external trauma, stapediovestibular dislocation is rare. In a recent study of stapediovestibular dislocation, most cases showed internal dislocation (depressed into the vestibule) and external dislocation was uncommon [4]. An internal dislocation can be caused by direct penetrating injury by cotton swabs or ear-pick, and external dislocation can occur when the annular ligament is torn by traumatic force. Comminuted fractures of the footplate can also cause stapediovestibular dislocation. In general, stapediovestibular dislocation causes PLF, and patients may complain of various cochleovestibular symptoms such as sensorineural hearing loss, mixed hearing loss, and vertigo as well as conductive hearing loss. PLF can be suspected if the patient has symptoms of vertigo and pneumolabyrinth is confirmed by imaging study [5]. If PLF has occurred but the patient has a loss of consciousness immediately after the accident or has more serious damage to other organs, the PLF may recover spontaneously and the patient may not be aware of the vestibular symptoms [3]. In our case, all types of ossicular dislocation were observed, including stapediovestibular dislocation. The patient might have had sensorineural hearing loss or severe dizziness, suggestive of PLF at the time of accident, but he had no memory of vestibulocochlear symptoms, possibly precluding a PLF. Management of stapediovestibular dislocation depends on the integrity and position of the stapes as well as the preoperative hearing status [4]. In cases in the literature, stapediovestibular dislocation was treated by various surgical methods, the most common of which was 'stapes repositioning and sealing' or stapedotomy/stapedectomy, and overall the hearing results were reported to be good $[4,5]$. In our case, stapes repositioning was not considered and sealing seemed to have occurred spontaneously a long time ago. Therefore, cartilage was reshaped to fit in the location of the missing footplate and the TORP was placed on it, which led to recovery of his hearing postoperatively.

In conclusion, our case involved long-standing external stapediovestibular dislocation with an oval window that had been completely replaced by fibrous tissue and was successfully treated with surgery that yielded a satisfactory hearing outcome.

\section{Acknowledgments}

This work was supported by research fund of Chungnam National University (BJK).

\section{Conflicts of interest}

The authors have no financial conflicts of interest.

\section{Author Contributions}

Conceptualization: Bong Jik Kim. Data curation: Geonho Lee and Bong Jik Kim. Formal analysis: Geonho Lee and Bong Jik Kim. Funding acquisition: Bong Jik Kim. Investigation: Bong Jik Kim. Methodology: Geonho Lee. Project administration: Geonho Lee. Resources: Yoonjoong Kim. Software: Geonho Lee. Supervision: Bong Jik Kim. Validation: Yoonjoong Kim. Visualization: Geonho Lee and Bong Jik Kim. Writing — original draft: Geonho Lee and Bong Jik Kim. Writing — review \& editing: Yoonjoong Kim and Bong Jik Kim. Approval of final manuscript: all authors.

\section{ORCID iDs}

Geonho Lee

Yoonjoong Kim

https://orcid.org/0000-0002-9531-1471

Bong Jik Kim

https://orcid.org/0000-0002-9589-9244

https://orcid.org/0000-0002-6384-2171

\section{REFERENCES}

1) Meriot $P$, Veillon F, Garcia JF, Nonent M, Jezequel J, Bourjat $P$, et al. CT appearances of ossicular injuries. Radiographics 1997;17:144554.

2) Hakuba N, Iwanaga M, Tanaka S, Hiratsuka Y, Kumabe Y, Konishi M, et al. Ear-pick injury as a traumatic ossicular damage in Japan. Eur Arch Otorhinolaryngol 2010;267:1035-9.

3) Yetiser S, Hidir Y, Birkent H, Satar B, Durmaz A. Traumatic ossicular dislocations: etiology and management. Am J Otolaryngol 2008; 29:31-6.

4) Bogaerts M, Waterval J, van Dinther J, Somers T, Zarowski A, Offeciers FE. Treatment of traumatic stapediovestibular luxation: case report with the introduction of a new technique and review of literature. Otol Neurotol 2014;35:582-8.

5) Delrue S, Verhaert N, Dinther JV, Zarowski A, Somers T, Desloovere C, et al. Surgical management and hearing outcome of traumatic ossicular injuries. J Int Adv Otol 2016;12:231-6. 